\title{
Ankle Hindfoot Scale vs Comprehensive SRMC Scoring System in Acute Achilles Tendon Rupture
}

\author{
1Jambu Nageswaran, ${ }^{2}$ Ganesan Ganesan Ram, ${ }^{3}$ Samuel Chittaranjan Bedford
}

\begin{abstract}
The existing scoring system like American Orthopaedic Foot and Ankle Society (AOFAS)-ankle hindfoot scale, Leppilahti et al scoring system, Achilles tendon total rupture score, foot and ankle outcome score, the physical activity scale (PAS) questionnaire and Tegner activity score were not designed to evaluate all the parameters following surgical treatment of Achilles tendon rupture. Hence, we developed a comprehensive score called the comprehensive Sri Ramachandra Medical College (SRMC) scoring system. We compared the results of 60 patients who were surgically treated for acute Achilles tendon rupture using our comprehensive SRMC scoring system with the most commonly used AOFAS-ankle hindfoot scale.
\end{abstract}

Results: We found that there was $6.7 \%$ poor results using our scoring system, whereas no poor results using AOFAS scoring system mainly because our scoring system also assesses quality of life, patient satisfaction and tendon strength. Similarly, there was $10 \%$ fair results in our scoring system compared to $3.3 \%$ in AOFAS scale indicating that our scoring system is more sensitive. Comprehensive SRMC scoring system was found to be reliable statistically as per alpha method $(\alpha=0.7787)$ and was statistically significant at $p<0.001$.

Conclusion: Comprehensive SRMC scoring system was found to be superior to AOFAS scale specifically for analyzing Achilles tendon repair. Poor results are likely to be picked up by our scoring system which has been shown in our results.

Keywords: Acute Achilles tendon rupture, Parameters of AOFAS-Ankle hindfoot scale, Parameters of comprehensive SRMC scoring system.

How to cite this article: Nageswaran J, Ram GG, Bedford SC. Ankle Hindfoot Scale vs Comprehensive SRMC Scoring System in Acute Achilles Tendon Rupture. J Foot Ankle Surg (AsiaPacific) 2015;2(2):77-79.

Source of support: Nil

Conflict of interest: None

\section{INTRODUCTION}

The Achilles tendon injury can be quite disabling. Knowing whether a given treatment or surgical technique

\footnotetext{
${ }^{1,3}$ Professor, ${ }^{2}$ Associate Professor

${ }^{1-3}$ Department of Orthopedics, Sri Ramachandra University Chennai, Tamil Nadu, India

Corresponding Author: Jambu Nageswaran, Professor Department of Orthopedics, Sri Ramachandra University Chennai, Tamil Nadu, India, Phone: 04445928541, e-mail: doctorjambu@gmail.com
}

results in full recovery of function and restoring back good quality of life is important. ${ }^{1}$ The great challenge for researchers lies in following specific scoring system for specific injury. In this article, we assessed whether comprehensive SRMC scoring system is better than ankle hindfoot scoring system.

\section{AIM}

To compare ankle hindfoot scale of American Orthopaedic Foot and Ankle Society (AOFAS) scoring system with comprehensive SRMC scoring system in the management of acute Achilles tendon rupture.

\section{MATERIALS AND METHODS}

This is a prospective study done in Department of Orthopedics, Sri Ramachandra Medical College, Chennai, India. The study period was from Jan 2012 to Jan 2015. The inclusion criterion was acute Achilles tendon rupture patients above 18 years including partial tendon injuries with at least 1 year follow-up. The exclusion criteria were chronic rupture including spontaneous Achilles tendon rupture, closed ruptures and patients who underwent revision Achilles tendon repair. After obtaining institutional ethics committee approval 62 patients were included in the study. Two patients lost follow-up and only 60 patients completed the study. All 60 patients had open wounds. All the patients were clearly explained about the study in their mother tongue (Tamil) and informed consent obtained. Patient's follow-up was done using both ankle hindfoot score ${ }^{2}$ and comprehensive SRMC scoring system. ${ }^{3}$

All the patients had a detailed history and physical exam. Thompson test (Simmond's squeeze test), decreased ankle plantar flexion strength, presence of a palpable gap (defect, loss of contour) and increased passive ankle dorsiflexion with gentle manipulation ${ }^{4}$ were used to diagnose closed rupture. The average age was 40 years and $80 \%$ of mode of injury was traumatic including sharp cut injuries $(40 \%)$ and toilet injuries $(40 \%)$. Since all of the patients had open injuries, ruptured tendon was clinically visualized and palpated. All the surgeries were done within 24 hours. All the open wound patients underwent emergency wound debridement and primary repair of Achilles tendon. All patients 
underwent primary end to end repair with either Bunell's or Kessler's stitch. ${ }^{5,6}$ Postoperatively patients were put on above knee POP slab for 2 weeks with ankle in equinus and knee in $30^{\circ}$ flexion. After suture removal at the end of 2 weeks slab was converted into below knee cast with ankle in equinus for 2 more weeks. Followed by below knee walking POP in neutral position with weight bearing for 2 more weeks. Average period of ankle immobilization was 7 weeks. The period of immobilization varied with the merit of the case. All patients were followed at regular intervals up to 1 year. The statistical analysis was done using paired sample test.

\section{RESULTS}

We had 97\% excellent/good results using ankle hindfoot scoring system and $83 \%$ good results as per comprehensive SRMC scoring. The comparisons of the results were tabulated in Table 1. Eight patients had sural nerve hypothesia and calf atrophy was noted in nine patients. We had three cases of superficial infections, which settled down with antibiotics and regular dressings.

\section{DISCUSSION}

Achilles tendon is the thickest and strongest tendon in the human body. Despite its strength, it is susceptible to both overuse injury and acute injury, such as a complete rupture. The incidence of Achilles tendon rupture appears to be rising and approximately $75 \%$ of all ruptures occur during sporting activities. ${ }^{7}$ We have not included chronic and spontaneous Achilles tendon injuries as all these cases will have multiple comorbidity including diabetes mellitus, steroid intake and iatrogenic steroid injection into the tendon and these cases cannot be managed by end to end repair as they will have big gap rupture and will require some form of reconstruction and we also know that the healing potential of chronic and acute ruptures are different. Surgical management is the preferred choice of treatment. There are numerous scoring system used to assess the functional outcome of surgically treated Achilles tendon rupture. Some of them are Leppilahti et al scoring system, ${ }^{8}$ Achilles tendon total rupture score, ${ }^{9}$ foot and ankle outcome score, ${ }^{10}$ the physical activity scale (PAS) questionnaire $^{11}$ Tegner activity score, ${ }^{12}$ ankle hindfoot

Table 1: Comparison of results between ankle hindfoot scoring system and comprehensive SRMC scoring system

\begin{tabular}{llllll}
\hline & \multicolumn{2}{c}{ Ankle hindfoot scoring } & & \multicolumn{2}{l}{$\begin{array}{l}\text { Comprehensive SRMC } \\
\text { scoring }\end{array}$} \\
\cline { 2 - 3 } \cline { 5 - 6 } & Numbers & Percentage & & Numbers & Percentage \\
\hline Excellent & 46 & 72.9 & & - & - \\
Good & 12 & 19.8 & & 50 & 83.3 \\
Fair & 2 & 3.3 & & 06 & 10 \\
Poor & 0 & 0 & 04 & 6.7 \\
\hline
\end{tabular}

scoring system. Out of these scoring system ankle hindfoot scoring system was widely used. All these scoring systems have its own advantages and disadvantages. A successful scoring system should have validity, reliability, practicality and accuracy.

American Foot and Ankle Society devised ankle hindfoot scoring system. This scale is based upon factors like pain, alignment and function. The advantages were that it scale grades ankle, subtalar, talonavicular and calcaneocuboid joint levels. It can be used for various foot and ankle problem. Its demerits were patient's satisfaction, tendon strength and the quality of life following surgery was not discussed. Moreover, it is not specific for Achilles tendon. Various aspects, such as muscle strength and endurance, joint range of motion and tendon strength can affect lower leg function. These parameters were mandatory for any successful scoring system. Measurements, such as calf circumference, ankle range of motion, calf muscle strength and endurance and gait analysis should be taken into consideration while evaluating functional outcome after an Achilles tendon repair. To overcome shortcomings of other scoring system we evolved comprehensive SRMC scoring system, which will be specific for Achilles tendon rupture follow-up. The uses of reliable, valid and objective measurements were included in comprehensive SRMC scoring system.

The parameters that are considered are quality of life, tendon strength, wound healing, tendon geometry, pain, strength, gait, ankle range of movements, calf atrophy and patients satisfaction. We were able to pick-up $6.7 \%$ of poor results compared to ankle hindfoot scale of AOFAS which did not have any poor results only because we had parameters to assess quality of life, patient satisfaction and tendon strength (Fig. 1) which was very useful to any tendon repair. We have assessed the strength of the tendon clinically by assessing ability to stand with both the heel raised, ability to stand in single heel raise and hopping and jumping. We also devised our own apparatus

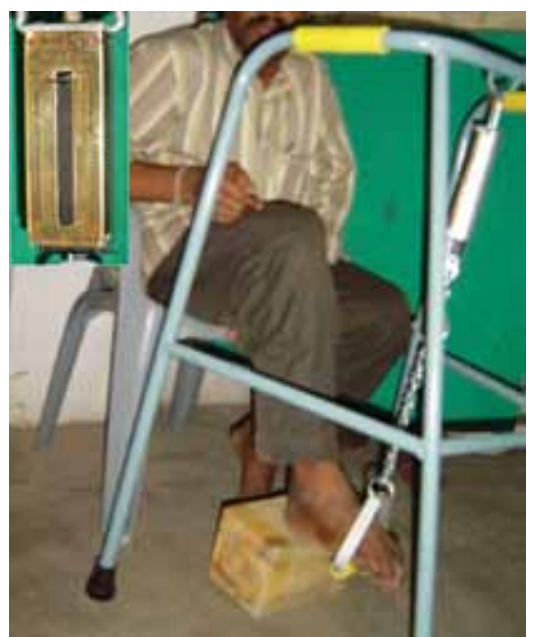

Fig. 1: Apparatus divised by us to assess tendon strength 
to assess the tendon strength postsurgically which was cost-effective and simple for people of low socioeconomic status (Fig. 1). Pain is more a subjective criteria and still 40 points were allotted to pain alone in AOFAS, whereas in our scoring system, we have given 10 points each to pain, quality of life and patient satisfaction. From Tables 2 and 3 , it is clearly evident that the comprehensive SRMC scoring system was found to be reliable statistically as per alpha method ( $\alpha=0.7787)$ and was statistically significant at $\mathrm{p}<0.001$.

\section{CONCLUSION}

Comprehensive SRMC scoring system includes more parameters for a sensitive scoring system, especially quality of life, tendon strength postsurgery and patient satisfaction which are not highlighted in AOFAS-ankle hindfoot scale. Moreover, it is specific for Achilles tendon injuries follow-up while ankle hindfoot scale is a generalized one for all ankle and hindfoot injuries. Our scoring system can also be extended to analyze surgically managed chronic and spontaneous Achilles tendon ruptures even though the healing potential is different. Though we do not have criteria in our scoring system

Table 2: Correlation

\begin{tabular}{llll}
\hline & & SRMC scores & AOFAS score \\
\hline Pearson's & SRMC score & 1.000 & 0.7666 \\
correlation & AOFAS score & 0.7666 & 1.000 \\
Sig. & SRMC score & - & 0.000 \\
(2 Tailed) & AOFAS score & 0.000 & - \\
\hline
\end{tabular}

Paired sample test at SS $p<0.001$

Table 3: Statistical analysis

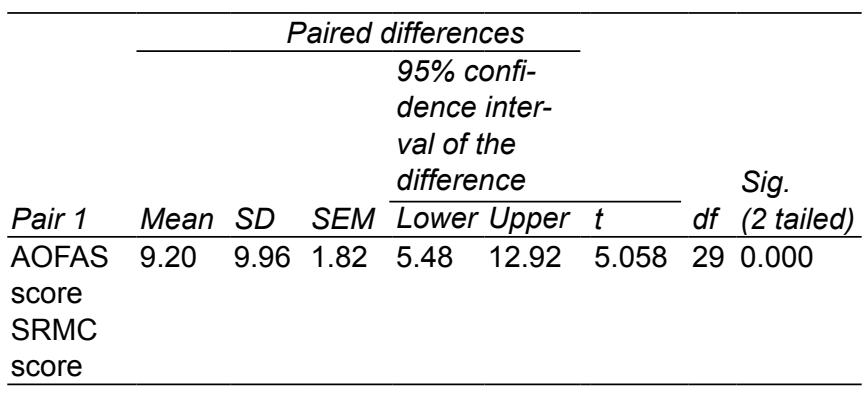

to differentiate excellent and good scores compared to AOFAS, still poor results cannot be missed by our scoring system which has been shown in our results. For evaluating Achilles tendon injury, comprehensive SRMC scoring system is better than ankle hindfoot scale of AOFAS.

\section{REFERENCES}

1. Nauck T, Lohrer H. Translation, cross-cultural adaption and validation of the german version of the foot and ankle ability measure for patients with chronic ankle instability. Br J Sports Med 45;10(2011):785.

2. Kitaoka HB. Clinical rating system for the ankle, hind foot, mid foot, hallux, and lesser toes. Foot Ankle J 1994 Jul;15(7): 349-353.

3. Nageshwaram J, Ganesan GR, Subburayan RB. Outcome analysis of surgically treated Tendo Achilles rupture. Int J Res Med Sci 2015;3(1):161-164.

4. Maffulli N. The clinical diagnosis of subcutaneous tear of the Achilles tendon: a prospective study in 174 patients. Am J Sports Med 1998;26(2):266-270.

5. McCoy BW, Haddad SL. The strength of Achilles tendon repair: a comparison of three suture techniques in human cadaver tendons. Foot Ankle Int 2010 Aug;31(8):701.

6. Lee YS, Lin CC, Chen CN, Chen SH, Liao WY, Huang CR. Reconstruction for neglected Achilles tendon rupture: the modified Bosworth technique. Orthoped 2005 Jul;28(7): 647-650.

7. Houshian S, Tscherning T, Riegels-Nielsen P. The epidemiology of Achilles tendon rupture in a Danish county. Injury 1998;29(9):651-654.

8. Leppilahti J, Forsman K, Puranen J, Orava S. Outcome and prognostic factors of Achilles rupture repair using a new scoring method. Clin Orthop Relat Res 1998 Jan;(346): 152-161.

9. Nilsson-Helander K, Thomee R, Gravare-Silbernagel K, et al. The Achilles tendon total fupture score (ATRS): development and validation. Am J Sports Med 2007;35(3):421-426.

10. Roos EM, Brandsson S, Karlsson J. Validation of the foot and ankle outcome score for ankle ligament reconstruction. Foot Ankle Int 2001;22(10):788-794.

11. Grimby G. Physical activity and muscle training in the elderly. Acta Med Scand Suppl 1986;711:233-237.

12. Ibrahim SA. Surgical treatment of chronic Achilles tendon rupture. J Foot Ankle Surg 2009 May-Jun;48(3):340-346. 